\title{
The impact of hypoxia on tumor-associated macrophages
}

\author{
Anne-Theres Henze ${ }^{1,2}$ and Massimiliano Mazzone $e^{1,2}$ \\ 'Laboratory of Tumor Inflammation and Angiogenesis, Vesalius Research Center, VIB, Leuven, Belgium. 'Laboratory of Tumor Inflammation and Angiogenesis, Department of Oncology, \\ KU Leuven, Leuven, Belgium.
}

\begin{abstract}
The role of tumor-associated macrophages (TAMs) in cancer is often correlated with poor prognosis, even though this statement should be interpreted with care, as the effects of macrophages primarily depend on their localization within the tumor. This versatile cell type orchestrates a broad spectrum of biological functions and exerts very complex and even opposing functions on cell death, immune stimulation or suppression, and angiogenesis, resulting in an overall pro- or antitumoral effect. We are only beginning to understand the environmental cues that contribute to transient retention of macrophages in a specific phenotype. It has become clear that hypoxia shapes and induces specific macrophage phenotypes that serve tumor malignancy, as hypoxia promotes immune evasion, angiogenesis, tumor cell survival, and metastatic dissemination. Additionally, TAMs in the hypoxic niches within the tumor are known to mediate resistance to several anticancer treatments and to promote cancer relapse. Thus, a careful characterization and understanding of this macrophage differentiation state is needed in order to efficiently tailor cancer therapy.
\end{abstract}

\section{Macrophages and the tumor microenvironment}

Tumor tissue comprises a highly complex network of diverse cell types. In a simplified view, the tumor microenvironment can be subdivided into the cancer cell and the stromal cell compartments, the latter of which harbors many different cell types. A considerable number of innate immune cells reside within the tumor microenvironment, including macrophages, which are the most prevalent cell type in a variety of tumors. Macrophages are primarily considered essential mediators in immune defense and it has been assumed that they might contribute to an antitumor immune response to combat tumor growth; however, mounting evidence indicates that macrophages foster malignancy and tumor progression. The discrepancy between these contrasting observations might stem from the fact that, in general, macrophages are exceptionally plastic cells that respond and adapt to the microenvironment in which they are embedded $(1,2)$. Moreover, the functional properties of tissue-resident macrophages greatly vary between different organs, not only due to their environmental skewing (3), but also with respect to their specific origin, further complicating a simplified classification of macrophages. For example, brain macrophages arise from the yolk sac (4), Kupffer cells are believed to arise from a mixed lineage that includes the yolk sac and embryonic hematopoietic stem cells (5), and macrophages in the intestine are derived entirely from adult hematopoietic stem cells (6). During adulthood each organ determines to what extent tissue-circulating blood monocytes replace tissue-resident macrophages. However, it still remains an open question as to what extent cell lineage determines macrophage behavior and phenotype (7). A recent study demonstrated for the first time that bone

Conflict of interest: The authors have declared that no conflict of interest exists. Reference information: / Clin Invest. 2016;126(10):3672-3679. doi:10.1172/JCI84427. marrow-derived macrophages can give rise to self-renewing tissue-resident macrophages (in this case, Kupffer cells) during the first weeks of life or during tissue injury in adulthood when the niche becomes available. These bone marrow-derived macrophages and their embryonic counterparts were further shown to exhibit a significant phenotypic and transcriptional overlap (8).

Macrophages are equipped to execute a broad repertoire of functions that range from their involvement in tissue homeostasis and wound healing to their role as immune effectors. In a simplified view, there are two main activation states that represent a paradigm for understanding the opposing functions that these cells can carry out: the classical M1 and the alternative M2 macrophage phenotypes, a classification that mirrors the Th1/Th2 polarization of $\mathrm{T}$ cells (9). Macrophage responses, which are shaped by these activation modes, are characterized by two contrasting actions: killing and repairing. Proinflammatory cytokines such as TNF- $\alpha$ or IFN- $\gamma$, as well as microbial cell wall components, serve as cues that trigger a proinflammatory, antibacterial, and antiangiogenic (M1-like) program, thereby arming macrophages with important effector molecules that allow pathogen recognition and killing as well as recruitment of other immune cells to the site of infection. Generation of ROS and NO, expression of high amounts of IL-12, and low levels of IL-10 are typically associated with an M1 macrophage response. In contrast, cytokines such as IL-4, IL-13, and IL-10 induce macrophages to acquire the ability to execute antiinflammatory, protumorigenic, and proangiogenic (M2-like) functions (10, 11). Under physiological conditions, M2-like macrophages facilitate wound healing by promoting angiogenesis, cell proliferation, and clearing of cellular debris $(12,13)$. However, as we will discuss in more detail, these capacities are coopted within the tumor microenvironment to fuel tumor growth. In reality, the distinction between these activation states becomes rather blurry, as macrophages might exhibit phenotypes anywhere in between these two extremes. Recent pub- 
lications dealing with this issue shed new light on the complexity of macrophage activation states (and ontogeny), proposing new guidelines for nomenclature and experimental standards to describe macrophage heterogeneity $(14,15)$.

Within the cancerous tissue, macrophages are designated as tumor-associated macrophages (TAMs). It is clear that M1-like macrophages can contribute to an antitumor response; however, experimental evidence suggests that during tumor initiation, persistent, proinflammatory (M1-like) macrophage functions might promote malignant transformation (16), supporting the hypothesis that inflammation promotes cancer, which was established by Rudolf Virchow in the $19^{\text {th }}$ century (17). An overwhelming and long-lasting release of inflammation-promoting factors, such as cytokines, chemokines, or free radicals, has been shown to culminate in cell proliferation, mutagenesis, and oncogene activation, all of which are determinants of cell transformation (16). Thus, chronic inflammatory conditions are characterized by a greater risk of tumor incidence. In an established tumor, macrophages are educated to a proangiogenic tumor-promoting (M2-like) phenotype. Tumor hypoxia is thought to play a pivotal role in the phenotypic control of TAMs, as hypoxic TAMs release factors that assist in tumor growth, cancer immunosuppression, and angiogenesis $(18,19)$. It is therefore easy to conceive that phenotypic switches in TAMs occur in a spatiotemporal fashion, as they are dependent on microenvironmental cues such as hypoxia in conjunction with cytokine availability. It should be noted that it is the concerted action of hypoxia and cytokines that ultimately shapes macrophage responses, as hypoxia alone is a common feature in many pathophysiological settings (20-22) in which the respective macrophage phenotype might differ strongly. LPS in sepsis is an M1 stimulus and hypoxic macrophages in sepsis will most likely possess an M1-like phenotype, whereas hypoxic TAMs are more strongly associated with an M2-like response (18), illustrating how a combination of factors ultimately directs the macrophage response.

\section{Tumor hypoxia}

Oxygen homeostasis is indispensable for the survival of all aerobic organisms. Molecular oxygen has evolved as the terminal electron acceptor in the mitochondrial electron transport chain - a deadly dependency, as every disturbance of oxygen availability endangers the respiratory capacity of the cell and, consequently, cellular survival (23). Thus, different mechanisms have evolved to restore oxygen homeostasis in order to protect against tissue damage (24, 25). Importantly, a considerable number of pathophysiological conditions are linked with tissue hypoxia, which frequently favors disease progression $(18,26)$.

Perturbation in oxygen homeostasis is a common feature of solid tumors, in which preclinical evidence has demonstrated that hypoxia correlates with a poor prognosis (27-29). However, in clinical settings the lack of an accurate and approved method to evaluate tumor hypoxia accounts for the limited capacity to intervene with a personalized hypoxia-based therapy. Thus, further studies are needed to determine if hypoxia can indeed serve as a reliable prognostic marker in clinical settings; contrary to the accepted opinion that hypoxia causes resistance to chemotherapy, some hypoxia-induced alterations might actually sensitize cells to chemotherapy (30).
Tumor hypoxia occurs when uncontrolled cell proliferation prevails such that blood vessel growth and the supply of oxygen and nutrition become limiting. The hypoxic response initiates a program to restore oxygen availability. On the cellular level this is reflected by angiogenesis induction, metabolic reprogramming, proliferation, self-renewal, and autophagy, which are among multiple mechanisms to counteract oxygen shortage; however, these are also detrimental processes that are exploited to foster tumor progression and metastatic dissemination (31). Therefore, the consequences of oxygen shortage are multifaceted, but exhibit a commonality in that they contribute to a hostile microenvironment that selects for a more aggressive cancer phenotype (32).

The hypoxic response is mediated by, among others, the hypoxia-inducible transcription factors HIF1 $\alpha$ and HIF2 $\alpha$, making it apparent how perturbations in oxygen availability can alter cellular responses. M1 and M2 macrophages differentially express HIF1 $\alpha$ and HIF $2 \alpha$ as well as inducible nitric oxide synthase (iNOS) and arginase 1 (ARG1) (33). Myeloid-specific loss of HIF1 $\alpha$ reduced tumor growth and retarded tumor progression in the murine MMTV-PyMT model (34). Loss of HIF2 $\alpha$ in macrophages had a favorable outcome in hepatocellular and colitis-associated colon carcinomas (35).

Under oxygen deprivation the angiogenic process is induced to ensure oxygen availability; however, the excessive release of angiogenic factors within the tumor microenvironment under hypoxic conditions culminates in a rather tortuous vascular network that does not effectively restore the blood supply. This aberrant vascular structure further contributes to spatiotemporal changes in oxygen delivery, thereby aggravating the hypoxic phenotype of tumors. Additionally, hypoxic cells are subjected to selection pressure, with the most aggressive cells surviving these hostile growth conditions and driving tumor growth (36). Oxygen shortage results in electron leakage and the generation of ROS, which subsequently oxidize proteins and cause DNA damage. As a net outcome, hypoxic cells experience genomic instability, which might further foster the accumulation of oncogenic drivers that then accelerate malignant progression (37).

From a therapeutic point of view, an inoperable vascular network limits the response to irradiation and reduces the efficacy of chemotherapeutics through different mechanisms such as insufficient distribution of the drug or a decrease in therapeutic cytotoxicity due to low oxygen levels, and/or the presence of a more acidic microenvironment (38-40).

A self-sustaining capacity of the tumor microenvironment also includes immune regulatory function, as suppression of the immune cell executer function and evasion of the immune response play a pivotal role in tumor progression (41). Hypoxia is intertwined in this response, as it contributes to a general shift from an antitumoral Th1-type response to a protumoral Th2-type response. Macrophages massively infiltrate tumor tissue and they are found in normoxic and hypoxic tumor compartments, albeit in different polarization states (42). The remainder of this review will focus on macrophages and elaborate on how hypoxia influences the TAM phenotype with respect to its contribution to disease progression.

\section{Protumoral function of hypoxic TAMs}

Double staining of hypoxia and macrophage markers reveals that macrophages massively infiltrate hypoxic/necrotic regions in 


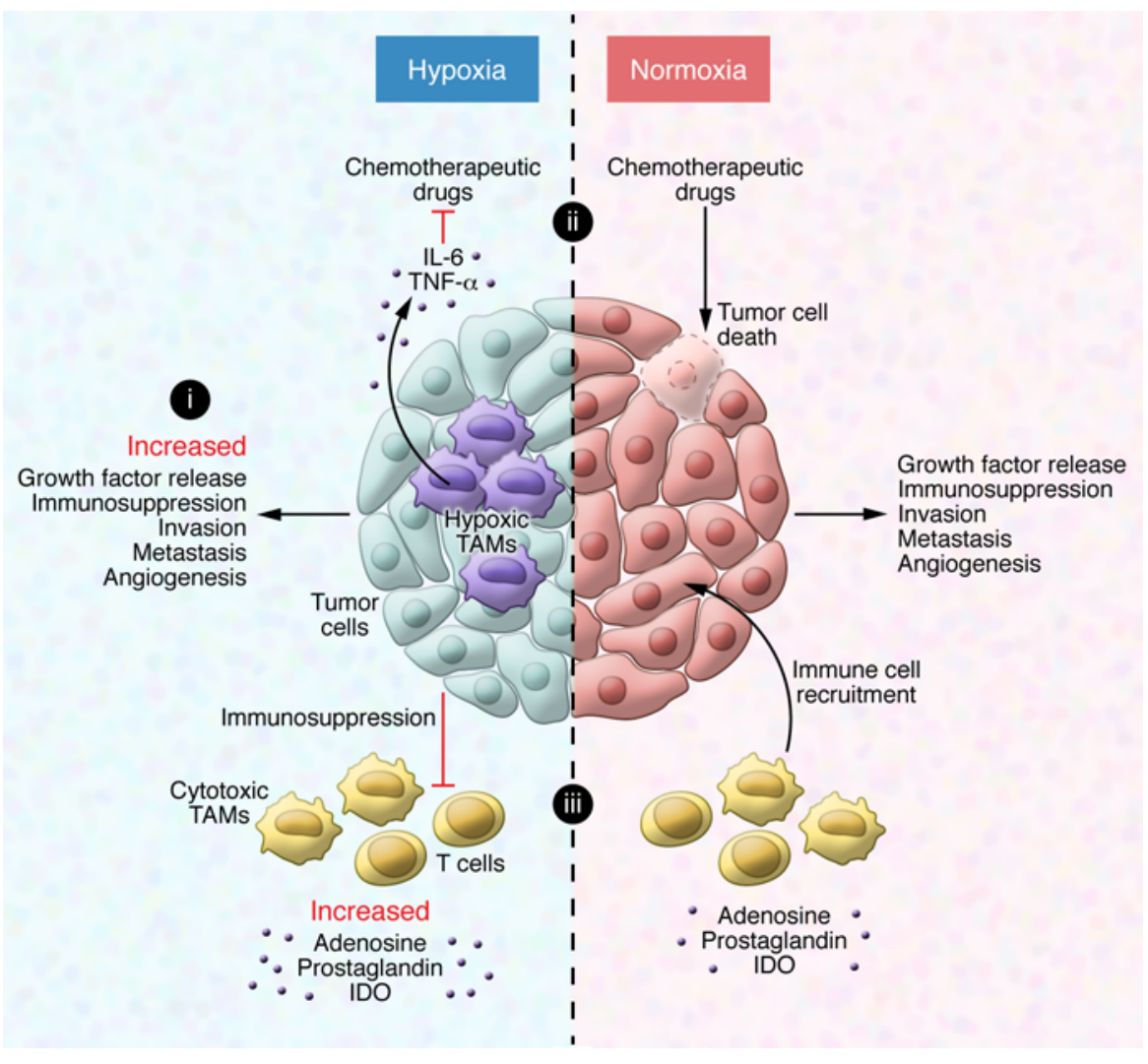

Figure 1. Hypoxic regulation of TAMs in cancer progression and therapy. (i) Hypoxia-induced release of chemoattractants results in enhanced TAM recruitment, which further amplifies the protumoral response. (ii) TAMs release survival factors for cancer cells, which protect them from chemotherapeutics. (iii) The hypoxic tumor environment is immunosuppressive and prevents an antitumor response. cooperation with an increasing gradient of migratory stimulating factors such as CCL2, CCL5, colony-stimulating factor 1 (CSF1, also known as macrophage colony-stimulating factor, $\mathrm{M}-\mathrm{CSF}$ ), VEGF, semaphorin 3A (SEMA3A), endothelial cell monocyte-activating polypeptide-II (EMAP-II), endothelin, stromal cell-derived factor $1 \alpha(\mathrm{SDF} 1 \alpha)$, eotaxin, and oncosatin M (19, 43-45). Hypoxia induces the expression of VEGF, EMAP-II, endothelin, SEMA3A, SDF1 $\alpha$, eotaxin, and oncosatin $\mathrm{M}$, thus explaining the enhanced availability of these factors within oxygen-deprived regions (19, 44-49). The cytokine-rich tumor microenvironment further provides factors such as CCL2, CCL5, or CSF1 produced by many different cell types such as tumor cells, fibroblasts, endothelial cells, or TAMs themselves (43). Apart from these chemoattractants, signals released from necrotic cells may also trigger the recruitment of innate immune cells through damage-associated molecular pattern (DAMP) receptors and macrophages to clear cellular debris (50, 51). Once macrophages arrive in these tumor compartments their mobility is slowed down via hypoxia-dependent mechanisms. In conjunction with the decrease in oxygen, macrophage expression of CCR2, CCR5, and neuropilin-1 (NRP1) is markedly diminished, disrupting these signaling pathways to halt macrophage mobility and trap TAMs within the hypoxic niche (19, 52, 53). Furthermore, MAPK phosphatase 1 (MPK1), which is upregulated under hypoxic conditions, dephosphorylates enzymes such as MEK, ERK1/2, and p38 MAPK, blocking pathways that are triggered by migrationstimulating factors $(54,55)$. Thus, two plausible mechanisms might account for the accumulation of macrophages in hypoxic/necrotic regions: (a) the attraction of macrophages by a cytokine gradient and (b) the hampered mobility of macrophages within these areas.
Once entrapped, macrophages are primed to serve protumoral functions (Figure 1). We have shown that impeding TAM entry into hypoxic tumor regions by genetically targeting Nrp1 prevents angiogenesis and cancer immune escape, providing evidence that hypoxia can fuel TAM-driven vessel formation and immunosuppression (19). The means by which hypoxic TAMs promote tumor progression include the upregulation of growth factors such as FGF2, PDGF, and VEGF, which support the growth of tumor cells in nutrient-deprived regions $(56,57)$. However, most of our knowledge concerning these factors stems from in vitro studies; thus, the in vivo situation still needs to be validated. Which growth factor predominates depends on the tumor type. For example, EGF acts in a paracrine manner to induce production of CSF1 in breast cancer, ensuring macrophage survival as well as stimulating the growth of tumor cells $(58,59)$. Macrophages release proteolytic enzymes that destroy the extracellular matrix, thereby contributing to cancer cell invasion. Depending on the specific microenvironmental cues, macrophages secrete a number of different matrix metalloproteinases (MMPs) (60). Hypoxic TAMs specifically release MMP7 (61), which enhances tumor cell migration and invasion. Within the hypoxic microenvironment tumor cells increase expression of endothelin 1 and 2 (62), which concomitantly stimulate the release of MMP2 and MMP9 from macrophages (63), further assisting an effective migratory response. Moreover, hypoxic macrophages achieve a proangiogenic response either by directly upregulating angiogenic molecules (VEGF, FGF2, CXCL8, IL-8, type I receptor for VEGF, angiopoietin) or through upregulation of angiogenic modulators (COX2, iNOS, MMP7) (64). 
The hypoxic tumor microenvironment plays a fundamental role in immune evasion (Figure 1). By releasing prostaglandin E2 and IL-10, TAMs create an immune-suppressive environment that halts the immune response through several mechanisms (65-67). The function of immune effector cells such as $\mathrm{T}$ cells is impaired through the release of these suppressive factors, but also through a diminished activation of T cells by TAMs. For example, IL- 6 and IL-10 induce expression of programmed death-ligand 1 (PD-L1) (68). Moreover HIF1 $\alpha$ directly induces PD-L1 expression on macrophages. PD-L1 subsequently inhibits $\mathrm{T}$ cell effector function by binding its receptor, programmed death-1 (PD-1), on T cells (69). Additionally, TAMs attract CCR4-expressing Tregs to the tumor through the secretion of cytokines such as CCL17 and CCL22 (70, 71). Notably, Treg enrichment correlates with reduced survival in ovarian cancer (70). Macrophages cocultured with hypoxic tumor cells upregulate indoleamine 2,3-dioxygenase (IDO) expression, resulting in inhibition of $\mathrm{T}$ cell proliferation and IFN- $\gamma$ production by $\mathrm{CD}^{+}$and $\mathrm{CD}^{+} \mathrm{T}$ cells. Moreover, CD $25^{+} \mathrm{Foxp}^{+}$Tregs increase when IDO-expressing macrophages are cocultured with $\mathrm{CD} 4^{+} \mathrm{CD} 25^{-}$effector T cells (72).

Further experimental evidence for the protumoral function of hypoxic macrophages stems from the findings that two different TAM subsets, which derive from the same Ly $6 \mathrm{C}^{\text {hi }}$ monocyte population, reside in different intratumoral regions (73). TAMs with low MHC-II expression (MHC-II ${ }^{\mathrm{lo}}$ ) populate hypoxic areas, whereas TAMs with high MHC-II expression (MHC-II ${ }^{\text {hi) }}$ populate normoxic areas. While hypoxia does not seem to influence differentiation of these two TAM subsets or MHC-II expression levels, hypoxia does influence the MHC-II ${ }^{\mathrm{lo}}$ macrophage phenotype by fine-tuning hypoxia-responsive genes that are involved in metabolism, angiogenesis, and metastasis, thereby fostering tumor progression (74). The underlying mechanism for this phenotypic switch in MHC-II ${ }^{\mathrm{lo}}$ compared to MHC-II ${ }^{\text {hi }}$ macrophages, as well as why MHC-II ${ }^{\text {lo }}$ macrophages specifically reside in hypoxic areas, remains an open question; nevertheless, the gene expression profile specifically affected in these macrophages by hypoxia, such as increased availability of VEGFA, lactate dehydrogenase A ( $L D H A)$, or urokinase-type plasminogen activator receptor (UPAR) underscores the contribution of MHC-II ${ }^{\mathrm{lo}}$ macrophages to tumor progression. Thus, it is very likely that the modulation of HIFs in these populations impinges on their specific phenotype, as all of these genes have been reported to be HIF targets (75-77).

\section{TAMs and cancer therapy}

Tumor cells located in hypoxic tumor areas undergo a selection process in order to survive adverse growth conditions. As a result, they display a more aggressive phenotype and may acquire resistance to radiotherapy (30), while irradiation results in demolished or even hypoxic tissue. Macrophages recruited to clear the damaged tissue might counterproductively be skewed within these hypoxic areas towards a tumor-promoting phenotype, contributing to tumor relapse (Figure 1). Moreover, a specific subset of M2-type macrophages (MRC1 ${ }^{+} \mathrm{TIE} 2^{\mathrm{hi}} \mathrm{CXCR} 4^{\text {hi }}$ ) accumulates in perivascular regions following chemotherapy and accounts for high VEGFA expression in these regions (78). Thus, it is conceivable that these macrophages might initiate an angiogenic response that restores tumor vascularization. In contrast, low-dose irradia- tion primes T cells towards an antitumor response. This is accomplished through antigen release by dying tumor cells (79), as well as through M1 skewing (specifically characterized by NO release) of TAMs, which subsequently enhances $\mathrm{T}$ effector cell recruitment into the tumor (80).

The protumoral phenotypic skewing of macrophages within the hypoxic/necrotic tumor areas is further supported by the finding that the number of macrophages within the hypoxic area correlates with a bad prognosis (81). TAMs localize in high numbers in hypoxic tumor regions $(18,82,83)$ and, in general, TAM abundance within the tumor is correlated with a poor prognosis in a variety of tumor types $(2,84)$. However, this statement cannot be applied universally, as in several cases it is important to discriminate the specific tumor niche where TAMs accumulate or to evaluate specific macrophage subtypes. For example, TAM infiltration at the invasive front (in colon cancer) or at the tumor nest (in gastric cancer) has been associated with a promising disease outcome (85-87). In lung cancer, staining for the M2-specific macrophage marker CD204, which is expressed on alternatively activated alveolar macrophages, and for CD68, a pan-macrophage marker, has shown that it is not the total number of TAMs but rather a specific macrophage subset that correlates with prognosis. In this setting, accumulation of CD2O $4^{+} \mathrm{M} 2$ macrophages correlated with a worse disease outcome, whereas the overall $\mathrm{CD} 8^{+}$macrophage number did not correlate with disease progression (88). Therefore, despite the fact that clodronatemediated macrophage depletion, which targets all macrophage subtypes as well as other phagocytic cells, is beneficial in several tumor models, a body of evidence shows that the cytotoxic ability of macrophages is actually very important to abate tumor growth $(80,89)$. Findings in the RIP1-Tag5 (RT5) mouse model of spontaneous pancreatic islet carcinogenesis demonstrate that disease outcome is determined by macrophage functionality rather than the presence or absence of macrophages (80). This conclusion is supported by a study showing that histidine-rich glycoprotein (HRG) inhibits tumor growth and metastasis in different murine tumor models by inducing macrophage polarization from an M2 to an M1 phenotype (90).

Based on the studies described above, repolarization of macrophages to an antitumor phenotype might serve as a new angle of attack for tumor therapy. However, this view might also be too simplified, as currently there is limited evidence that repolarization of macrophages towards a tumoricidal phenotype is always beneficial for disease outcome. Further investigation will be required to determine whether the hypoxic microenvironment does not overrule the antitumor function of macrophages once they are repolarized towards an M1-like phenotype. An elegant study using a CD40 agonist in a genetically engineered mouse model of pancreatic ductal adenocarcinoma (PDAC) supports the findings that the activation of macrophages via this pathway might be enough to initiate an antitumor effect without $\mathrm{CD} 4^{+}$and $\mathrm{CD} 8^{+} \mathrm{T}$ cells. This study defines the mechanisms underlying a clinical trial of a combined CD40 agonist treatment with gemcitabine chemotherapy in a small cohort of PDAC patients (91). However, it is not known if this is a tumor type-specific response. Another report in hepatocellular carcinoma further supports the notion that macrophages may be key players in the antitumor response. Ex vivo IL-12 treat- 
ment evoked intratumoral macrophage infiltration and a T celland NK cell-independent antitumor response (92).

Due to the limited success of conventional chemotherapeutics, which are mainly directed against cancer cells, other therapeutic interventions such as antiangiogenic agents or immune therapeutics have shifted the focus to cells in the stroma. Combined approaches utilizing both conventional chemotherapeutics and stroma-targeting agents appear to be superior to monotherapy. As outlined in more detail below, the beneficial effects of targeting multiple processes likely stem from different mechanisms of action, as well as changes in the nature of the tumor microenvironment. In vitro studies have already demonstrated that the presence of macrophages can account for resistance towards chemotherapeutics, enhancing cancer cell survival (93). Thus, it is likely that combinatory approaches will be successful in the clinic. Indeed, to allow chemotherapeutic regimens to reach their highest cytotoxic effect, macrophage functions conferring cancer cell resistance to these drugs should be disabled so that the chemotherapeutics can function without being detoxified by the surrounding TAMs.

The mechanisms by which macrophages participate in therapy resistance are not fully understood. The secretion of cytokines such as IL-6 activates STAT3 signaling in neighboring tumor cells and may promote survival; however, the link between TAMs, IL-6 secretion, and therapy resistance in vivo has not been investigated (93). In melanoma, macrophage-dependent TNF- $\alpha$ release was shown to protect tumor cells from MEK and BRAF inhibitors (ref. 94 and Figure 1). Moreover, chemotherapeutics can trigger the release of macrophage chemoattractants from tumor cells, thus recruiting more monocytes/macrophages into the tumor, which promote tumor progression. CSF1 regulates monocyte-to-macrophage differentiation as well as macrophage recruitment and proliferation (95). Agents targeting CSF1 or its receptor (CSF1R) are in clinical development; however, as a monotherapy, such agents will most likely not be sufficient, and several combinatory approaches have been investigated or are still under evaluation, including combinations of CSF1/CSF1R-targeting agents with chemotherapeutics (96-98), irradiation (99), immune check point inhibitors (100), and antiangiogenic agents (101-103), among others. In animal breast cancer models, anti-CSF1R treatment in combination with paclitaxel diminishes tumor growth and metastasis formation and shifts the tumor from a CD4 ${ }^{+} \mathrm{T}$ cell-enriched environment towards a CD8 ${ }^{+} \mathrm{T}$ cell-enriched environment (96).

There are several other examples of successful combinations of well-established treatment regimens with immunotherapy. In B16 melanoma and 9464D neuroblastoma, multidrug chemotherapy (vincristine, cyclophosphamide, and doxorubicin) combined with immunotherapy (anti-CD40 and cytosine-phosphateguanosine-containing oligodeoxynucleotide 1826 [CpG-ODN]) enhanced NO, IFN- $\gamma$, and IL-12p40 secretion by macrophages, leading to a strong antitumor response (104).

Other options for treatment have arisen from the observation that metabolic reprogramming can contribute to macrophage plasticity and function. An increase in glycolysis, reprogramming of the TCA cycle, and reduced oxidative phosphorylation can ultimately enhance formation of ROS and NO in order to promote a proinflammatory macrophage response. Moreover, an amplified arginosuccinate/citrulline/arginine cycle further contributes to a proinflammatory response (105). A recent report supports the idea that glycolytic cancer cells polarize TAMs to an M2 phenotype via their secretion of lactic acid (106). In a murine model of mammary carcinoma, treatment with zoledronic acid and subsequent repolarization of macrophages to an M1-like phenotype further highlights that a change in the polarization state can be achieved through very different mechanisms (107). Zoledronic acid is a nitrogen-containing bisphosphonate that inhibits farnesyl pyrophosphate (FPP) synthase, an enzyme in the mevalonate pathway. This decreases the prenylation of GTPase signaling proteins, thereby altering cellular function $(108,109)$. We are only beginning to understand how metabolic reprogramming in macrophages can impact macrophage plasticity and function, and future studies on these aspects will allow new treatment options for cancer therapy.

Overall, the lack of an approved method to measure intratumoral hypoxia still represents an obstacle to better categorization and characterization of hypoxic TAMs in cancer patients. During the last several years, tumor specimens have been characterized with different macrophage markers and inconsistent analytical methods, which has led to confusion about the role of TAMs in tumors. Too little is known about the specific role of the hypoxic subtype in tumor progression, even though hypoxia is likely to markedly impact disease outcomes. For example, the immunosuppressive signaling molecule adenosine controls macrophage functionality. Hypoxia induces adenosine signaling via different mechanisms. HIF1 $\alpha$ transcriptionally upregulates the adenosine receptor A2B (110), thereby enhancing adenosine signaling under hypoxic conditions. Moreover, oxygen deprivation increases the extracellular conversion of ATP to adenosine by CD39 and CD73 $(111,112)$ and enhances ATP availability (113-116). Adenosinemediated immunosuppression seems to be a protective feedback mechanism to counter the hypoxia-driven inflammatory response in many pathological conditions. Regarding macrophages, adenosine both halts the proinflammatory cytokine release of M1 macrophages via A2A and A2B receptors (117) and enhances the release of the antiinflammatory cytokine IL-10, while M2 macrophages exhibit enhanced activation in the presence of adenosine mediated by increased expression of arginase-1, tissue inhibitor of metalloproteinase-1 (TIMP-1), and macrophage galactosetype C-type lectin-1 (Mgl-1) (118). With respect to its therapeutic relevance, adenosine also augments VEGF expression in an A2A receptor-dependent manner, enhancing tumor vascularization (119). Thus, apart from its well-known inhibitory function on effector T cells and NK cells, adenosine fuels protumor activities of macrophages (Figure 1). Further studies are needed to dissect the specific roles of the respective adenosine receptors in tumor growth in order to design new treatment strategies.

\section{Conclusion}

Hypoxic TAMs play a prominent role in tumorigenesis and could potentially serve as a new therapeutic target for cancer therapy. Because macrophages are a very heterogeneous population that encompasses different phenotypes, it is necessary to thoroughly characterize the specific macrophage polarization states in different tumor types and at different time points of cancer progression, 
and to determine how polarization state affects cross-talk with the other tumor components, including cancer cells, T cells, and endothelial cells. Macrophages are important gatekeepers in the immune response; however, during tumor progression they support processes that promote tumor growth and ultimately destroy the organism. Tumors are referred to as wounds that do not heal (120) because they are characterized by persistent inflammation; thus, disrupting inflammation will be critical to eliminating tumors. During wound healing, M2 macrophages are only transiently present, whereas they are persistently abundant in the tumor, possibly explaining why these wounds cannot heal. The immune system is equipped with highly plastic macrophages in order to balance the immune response such that an overwhelming proinflammatory response can be countered by an antiinflammatory response. As this balancing act is accomplished by diverse polarization/differentiation states of the same cell type, it is conceivable that broad depletion of macrophages might not be favorable; rather, phenotypic conversion of macrophages may be beneficial. Thus, a close characterization of macrophage diversity within specific microenvironments might offer important information for new treatment regimens.

\section{Acknowledgments}

A.T. Henze was supported by Fonds Wetenschappelijk Onderzoek (FWO) number $1297115 \mathrm{~N}$ and M. Mazzone is supported by a European Research Council (ERC) starting grant and a Worldwide Cancer Research grant (13-1031).

Address correspondence to: Massimiliano Mazzone or AnneTheres Henze, VIB Vesalius Research Center, Department of Oncology, University of Leuven, Campus Gasthuisberg, Herestraat 49, Box 912, B-3000 Leuven, Belgium. Phone: 32.16.37.32.13; E-mail: massimiliano.mazzone@vib-kuleuven.be (M. Mazzone), annetheres.henze@vib-kuleuven.be (A.T. Henze).
1. Sica A, Mantovani A. Macrophage plasticity and polarization: in vivo veritas. J Clin Invest. 2012;122(3):787-795.

2. Pollard JW. Tumour-educated macrophages promote tumour progression and metastasis. Nat Rev Cancer. 2004;4(1):71-78.

3. Gosselin D, et al. Environment drives selection and function of enhancers controlling tissue-specific macrophage identities. Cell. 2014;159(6):1327-1340.

4. Ginhoux F, et al. Fate mapping analysis reveals that adult microglia derive from primitive macrophages. Science. 2010;330(6005):841-845.

5. Schulz C, et al. A lineage of myeloid cells independent of Myb and hematopoietic stem cells. Science. 2012;336(6077):86-90.

6. Bain CC, et al. Resident and pro-inflammatory macrophages in the colon represent alternative context-dependent fates of the same Ly6Chi monocyte precursors. Mucosal Immunol. 2013;6(3):498-510.

7. Epelman S, Lavine KJ, Randolph GJ. Origin and functions of tissue macrophages. Immunity. 2014;41(1):21-35.

8. Scott CL, et al. Bone marrow-derived monocytes give rise to self-renewing and fully differentiated Kupffer cells. Nat Commun. 2016;7:10321.

9. Mills CD, Kincaid K, Alt JM, Heilman MJ, Hill AM. M-1/M-2 macrophages and the Th1/Th2 paradigm. JImmunol. 2000;164(12):6166-6173.

10. Gordon S. Alternative activation of macrophages. Nat Rev Immunol. 2003;3(1):23-35.

11. Mantovani A, Sica A, Sozzani S, Allavena P, Vecchi A, Locati M. The chemokine system in diverse forms of macrophage activation and polarization. Trends Immunol. 2004;25(12):677-686.

12. Leibovich SJ, Ross R. The role of the macrophage in wound repair. A study with hydrocortisone and antimacrophage serum. Am J Pathol. 1975;78(1):71-100.

13. Polverini PJ, Cotran PS, Gimbrone MA, Unanue ER. Activated macrophages induce vascular proliferation. Nature. 1977;269(5631):804-806.

14. Murray PJ, et al. Macrophage activation and polarization: nomenclature and experimental guidelines. Immunity. 2014;41(1):14-20.
15. Xue J, et al. Transcriptome-based network analysis reveals a spectrum model of human macrophage activation. Immunity. 2014;40(2):274-288.

16. Elinav E, Nowarski R, Thaiss CA, Hu B, Jin C, Flavell RA. Inflammation-induced cancer: crosstalk between tumours, immune cells and microorganisms. Nat Rev Cancer. 2013;13(11):759-771.

17. Virchow R. An address on the value of pathological experiments. Br Med J. 1881;2(1075):198-203.

18. Murdoch C, Lewis CE. Macrophage migration and gene expression in response to tumor hypoxia. Int J Cancer. 2005;117(5):701-708.

19. Casazza A, et al. Impeding macrophage entry into hypoxic tumor areas by Sema3A/Nrp1 signaling blockade inhibits angiogenesis and restores antitumor immunity. Cancer Cell. 2013;24(6):695-709.

20. Bazan NG, Palacios-Pelaez R, Lukiw WJ. Hypoxia signaling to genes: significance in Alzheimer's disease. Mol Neurobiol. 2002;26(2-3):283-298.

21. Bergeron M, Yu AY, Solway KE, Semenza GL, Sharp FR. Induction of hypoxia-inducible factor-1 (HIF-1) and its target genes following focal ischaemia in rat brain. Eur J Neurosci. 1999;11(12):4159-4170.

22. Eltzschig HK, Carmeliet P. Hypoxia and inflammation. N Engl J Med. 2011;364(7):656-665.

23. Guzy RD, Schumacker PT. Oxygen sensing by mitochondria at complex III: the paradox of increased reactive oxygen species during hypoxia. Exp Physiol. 2006;91(5):807-819.

24. Peers C. Oxygen-sensitive ion channels. Trends Pharmacol Sci. 1997;18(11):405-408.

25. Semenza GL. HIF-1: mediator of physiological and pathophysiological responses to hypoxia. JAppl Physiol. 2000;88(4):1474-1480.

26. Graeber TG, et al. Hypoxia-mediated selection of cells with diminished apoptotic potential in solid tumours. Nature. 1996;379(6560):88-91.

27. Leite de Oliveira R, et al. Gene-targeting of Phd 2 improves tumor response to chemotherapy and prevents side-toxicity. Cancer Cell. 2012;22(2):263-277.

28. Mazzone M, et al. Heterozygous deficiency of PHD2 restores tumor oxygenation and inhibits metastasis via endothelial normalization. Cell. 2009;136(5):839-851.
29. Pàez-Ribes M, et al. Antiangiogenic therapy elicits malignant progression of tumors to increased local invasion and distant metastasis. Cancer Cell. 2009;15(3):220-231.

30. Wilson WR, Hay MP. Targeting hypoxia in cancer therapy. Nat Rev Cancer. 2011;11(6):393-410.

31. Semenza GL. Defining the role of hypoxia-inducible factor 1 in cancer biology and therapeutics. Oncogene. 2010;29(5):625-634.

32. Harris AL. Hypoxia - a key regulatory factor in tumour growth. Nat Rev Cancer. 2002;2(1):38-47.

33. Takeda $\mathrm{N}$, et al. Differential activation and antagonistic function of HIF- $\alpha$ isoforms in macrophages are essential for NO homeostasis. Genes Dev. 2010;24(5):491-501.

34. Doedens AL, et al. Macrophage expression of hypoxia-inducible factor- $1 \alpha$ suppresses T-cell function and promotes tumor progression. Cancer Res. 2010;70(19):7465-7475.

35. Imtiyaz HZ, et al. Hypoxia-inducible factor $2 \alpha$ regulates macrophage function in mouse models of acute and tumor inflammation. JClin Invest. 2010;120(8):2699-2714.

36. Carmeliet P, Jain RK. Angiogenesis in cancer and other diseases. Nature. 2000;407(6801):249-257.

37. Sabharwal SS, Schumacker PT. Mitochondrial ROS in cancer: initiators, amplifiers or an Achilles' heel? Nat Rev Cancer. 2014;14(11):709-721.

38. Manallack DT. The $\mathrm{pK}(\mathrm{a})$ distribution of drugs: application to drug discovery. Perspect Medicin Chem. 2008;1:25-38.

39. Minchinton AI, Tannock IF. Drug penetration in solid tumours. Nat Rev Cancer. 2006;6(8):583-592.

40. Trédan O, Galmarini CM, Patel K, Tannock IF. Drug resistance and the solid tumor microenvironment. J Natl Cancer Inst. 2007;99(19):1441-1454.

41. Barsoum IB, Koti M, Siemens DR, Graham CH. Mechanisms of hypoxia-mediated immune escape in cancer. Cancer Res. 2014;74(24):7185-7190.

42. Biswas SK, Sica A, Lewis CE. Plasticity of macrophage function during tumor progression: regulation by distinct molecular mechanisms. J Immunol. 2008;180(4):2011-2017.

43. Murdoch C, Giannoudis A, Lewis CE. Mechanisms regulating the recruitment of macrophages into hypoxic areas of tumors and other ischemic 
tissues. Blood. 2004;104(8):2224-2234.

44. Tripathi C, et al. Macrophages are recruited to hypoxic tumor areas and acquire a pro-angiogenic M2-polarized phenotype via hypoxic cancer cell derived cytokines Oncostatin M and Eotaxin. Oncotarget. 2014;5(14):5350-5368.

45. Du R, et al. HIF1alpha induces the recruitment of bone marrow-derived vascular modulatory cells to regulate tumor angiogenesis and invasion. Cancer Cell. 2008;13(3):206-220.

46. Leek RD, Hunt NC, Landers RJ, Lewis CE, Royds JA, Harris AL. Macrophage infiltration is associated with VEGF and EGFR expression in breast cancer. J Pathol. 2000;190(4):430-436.

47. Grimshaw MJ, Wilson JL, Balkwill FR. Endothelin-2 is a macrophage chemoattractant: implications for macrophage distribution in tumors. Eur J Immunol. 2002;32(9):2393-2400.

48. Matschurat S, et al. Regulation of EMAP II by hypoxia. Am J Pathol. 2003;162(1):93-103.

49. Lewis JS, Landers RJ, Underwood JC, Harris AL, Lewis CE. Expression of vascular endothelial growth factor by macrophages is up-regulated in poorly vascularized areas of breast carcinomas. J Pathol. 2000;192(2):150-158.

50. Zhang X, Mosser DM. Macrophage activation by endogenous danger signals. J Pathol. 2008;214(2):161-178.

51. Soehnlein O, Lindbom L. Phagocyte partnership during the onset and resolution of inflammation. Nat Rev Immunol. 2010;10(6):427-439.

52. Bosco MC, Reffo G, Puppo M, Varesio L. Hypoxia inhibits the expression of the CCR5 chemokine receptor in macrophages. Cell Immunol. 2004;228(1):1-7.

53. Sica A, et al. Defective expression of the monocyte chemotactic protein-1 receptor CCR2 in macrophages associated with human ovarian carcinoma. JImmunol. 2000;164(2):733-738.

54. Sun H, Charles CH, Lau LF, Tonks NK. MKP-1 (3CH134), an immediate early gene product, is a dual specificity phosphatase that dephosphorylates MAP kinase in vivo. Cell. 1993;75(3):487-493.

55. Franklin CC, Kraft AS. Conditional expression of the mitogen-activated protein kinase (MAPK) phosphatase MKP-1 preferentially inhibits $\mathrm{p} 38$ MAPK and stress-activated protein kinase in U937 cells. J Biol Chem. 1997;272(27):16917-16923.

56. Kuwabara K, et al. Hypoxia-mediated induction of acidic/basic fibroblast growth factor and platelet-derived growth factor in mononuclear phagocytes stimulates growth of hypoxic endothelial cells. Proc Natl Acad Sci U S A. 1995;92(10):4606-4610.

57. Harmey JH, Dimitriadis E, Kay E, Redmond HP, Bouchier-Hayes D. Regulation of macrophage production of vascular endothelial growth factor (VEGF) by hypoxia and transforming growth factor $\beta$-1. Ann Surg Oncol. 1998;5(3):271-278.

58 . Wyckoff J, et al. A paracrine loop between tumor cells and macrophages is required for tumor cell migration in mammary tumors. Cancer Res. 2004;64(19):7022-7029.

59. Goswami S, et al. Macrophages promote the invasion of breast carcinoma cells via a colony-stimulating factor-1/epidermal growth factor paracrine loop. Cancer Res. 2005;65(12):5278-5283.

60. Lewis CE, Pollard JW. Distinct role of macro- phages in different tumor microenvironments. Cancer Res. 2006;66(2):605-612.

61. Burke B, et al. Hypoxia-induced gene expression in human macrophages: implications for ischemic tissues and hypoxia-regulated gene therapy. Am J Pathol. 2003;163(4):1233-1243.

62. Grimshaw MJ, Naylor S, Balkwill FR. Endothelin-2 is a hypoxia-induced autocrine survival factor for breast tumor cells. Mol Cancer Ther. 2002;1(14):1273-1281.

63. Grimshaw MJ, Hagemann T, Ayhan A, Gillett CE, Binder C, Balkwill FR. A role for endothelin-2 and its receptors in breast tumor cell invasion. Cancer Res. 2004;64(7):2461-2468.

64. White JR, et al. Genetic amplification of the transcriptional response to hypoxia as a novel means of identifying regulators of angiogenesis. Genomics. 2004;83(1):1-8.

65. Ertel W, Singh G, Morrison MH, Ayala A, Chaudry IH. Chemically induced hypotension increases PGE2 release and depresses macrophage antigen presentation. Am J Physiol. 1993;264(4 pt 2):R655-R660.

66. Alleva DG, Burger CJ, Elgert KD. Tumor growth increases Ia- macrophage synthesis of tumor necrosis factor-alpha and prostaglandin E2: changes in macrophage suppressor activity. J Leukoc Biol. 1993;53(5):550-558.

67. Sica A, et al. Autocrine production of IL-10 mediates defective IL-12 production and NF-kappa B activation in tumor-associated macrophages. Jimmunol. 2000;164(2):762-767.

68. Kryczek I, et al. B7-H4 expression identifies a novel suppressive macrophage population in human ovarian carcinoma. J Exp Med. 2006;203(4):871-881.

69. Kuang DM, et al. Activated monocytes in peritumoral stroma of hepatocellular carcinoma foster immune privilege and disease progression through PD-L1. JExp Med. 2009;206(6):1327-1337.

70. Curiel TJ, et al. Specific recruitment of regulatory $\mathrm{T}$ cells in ovarian carcinoma fosters immune privilege and predicts reduced survival. Nat Med. 2004;10(9):942-949.

71. Mizukami Y, et al. CCL17 and CCL22 chemokines within tumor microenvironment are related to accumulation of Foxp $3^{+}$regulatory T cells in gastric cancer. Int J Cancer. 2008;122(10):2286-2293.

72. Ye LY, et al. Hypoxia-induced epithelial-to-mesenchymal transition in hepatocellular carcinoma induces an immunosuppressive tumor microenvironment to promote metastasis. Cancer Res. 2016;76(4):818-830.

73. Movahedi K, et al. Different tumor microenvironments contain functionally distinct subsets of macrophages derived from Ly6C(high) monocytes. Cancer Res. 2010;70(14):5728-5739.

74. Laoui D, et al. Tumor hypoxia does not drive differentiation of tumor-associated macrophages but rather fine-tunes the M2-like macrophage population. Cancer Res. 2014;74(1):24-30.

75. Forsythe JA, et al. Activation of vascular endothelial growth factor gene transcription by hypoxia-inducible factor 1. Mol Cell Biol. 1996;16(9):4604-4613.

76. Firth JD, Ebert BL, Ratcliffe PJ. Hypoxic regulation of lactate dehydrogenase A. Interaction between hypoxia-inducible factor 1 and cAMP response elements. J Biol Chem 1995;270(36):21021-21027.

77. Büchler P, et al. Transcriptional regulation of urokinase-type plasminogen activator receptor by hypoxia-inducible factor 1 is crucial for invasion of pancreatic and liver cancer. Neoplasia. 2009;11(2):196-206.

78. Hughes R, et al. Perivascular M2 macrophages stimulate tumor relapse after chemotherapy. Cancer Res. 2015;75(17):3479-3491.

79. Tesniere A, et al. Immunogenic cancer cell death: a key-lock paradigm. Curr Opin Immunol. 2008;20(5):504-511.

80. Klug F, et al. Low-dose irradiation programs macrophage differentiation to an $\mathrm{iNOS}^{+} / \mathrm{M} 1$ phenotype that orchestrates effective T cell immunotherapy. Cancer Cell. 2013;24(5):589-602.

81. Ohno S, et al. Correlation of histological localization of tumor-associated macrophages with clinicopathological features in endometrial cancer. Anticancer Res. 2004;24(5C):3335-3342.

82. Onita T, et al. Hypoxia-induced, perinecrotic expression of endothelial Per-ARNT-Sim domain protein-1/hypoxia-inducible factor- $2 \alpha$ correlates with tumor progression, vascularization, and focal macrophage infiltration in bladder cancer. Clin Cancer Res. 2002;8(2):471-480.

83. Leek RD, Landers RJ, Harris AL, Lewis CE. Necrosis correlates with high vascular density and focal macrophage infiltration in invasive carcinoma of the breast. Br J Cancer. 1999;79(5-6):991-995.

84. Allavena P, Mantovani A. Immunology in the clinic review series; focus on cancer: tumourassociated macrophages: undisputed stars of the inflammatory tumour microenvironment. Clin Exp Immunol. 2012;167(2):195-205.

85. Forssell J, Oberg A, Henriksson ML, Stenling R, Jung A, Palmqvist R. High macrophage infiltration along the tumor front correlates with improved survival in colon cancer. Clin Cancer Res. 2007;13(5):1472-1479.

86. Ohno S, et al. The degree of macrophage infiltration into the cancer cell nest is a significant predictor of survival in gastric cancer patients. Anticancer Res. 2003;23(6D):5015-5022.

87. Zhou Q, et al. The density of macrophages in the invasive front is inversely correlated to liver metastasis in colon cancer. J Transl Med. 2010;8:13.

88. Ohtaki Y, et al. Stromal macrophage expressing CD204 is associated with tumor aggressiveness in lung adenocarcinoma. J Thorac Oncol. 2010;5(10):1507-1515.

89. Oosterling SJ, et al. Macrophages direct tumour histology and clinical outcome in a colon cancer model. J Pathol. 2005;207(2):147-155.

90. Rolny C, et al. HRG inhibits tumor growth and metastasis by inducing macrophage polarization and vessel normalization through downregulation of PlGF. Cancer Cell. 2011;19(1):31-44.

91. Beatty GL, et al. CD40 agonists alter tumor stroma and show efficacy against pancreatic carcinoma in mice and humans. Science. 2011;331(6024):1612-1616.

92. Peron JM, et al. Treatment of murine hepatocellular carcinoma using genetically modified cells to express interleukin-12. J Gastroenterol Hepatol. 2004;19(4):388-396. 
93. Ruffell B, Coussens LM. Macrophages and therapeutic resistance in cancer. Cancer Cell. 2015;27(4):462-472.

94. Smith MP, et al. The immune microenvironment confers resistance to MAPK pathway inhibitors through macrophage-derived TNF $\alpha$. Cancer Discov. 2014;4(10):1214-1229.

95. Chitu V, Stanley ER. Colony-stimulating factor-1 in immunity and inflammation. Curr Opin Immunol. 2006;18(1):39-48.

96. DeNardo DG, et al. Leukocyte complexity predicts breast cancer survival and functionally regulates response to chemotherapy. Cancer Discov. 2011;1(1):54-67.

97. Ruffell B, et al. Macrophage IL-10 blocks CD8 ${ }^{+}$ $\mathrm{T}$ cell-dependent responses to chemotherapy by suppressing IL-12 expression in intratumoral dendritic cells. Cancer Cell. 2014;26(5):623-637.

98. Mitchem JB, et al. Targeting tumor-infiltrating macrophages decreases tumor-initiating cells, relieves immunosuppression, and improves chemotherapeutic responses. Cancer Res. 2013;73(3):1128-1141.

99. Xu J, et al. CSF1R signaling blockade stanches tumor-infiltrating myeloid cells and improves the efficacy of radiotherapy in prostate cancer. Cancer Res. 2013;73(9):2782-2794.

100.Zhu Y, et al. CSF1/CSF1R blockade reprograms tumor-infiltrating macrophages and improves response to T-cell checkpoint immunotherapy in pancreatic cancer models. Cancer Res. 2014;74(18):5057-5069.

101. Priceman SJ, et al. Targeting distinct tumor-infiltrating myeloid cells by inhibiting CSF-1 receptor: combating tumor evasion of antiangiogenic therapy. Blood. 2010;115(7):1461-1471.

102. Kubota Y, et al. M-CSF inhibition selectively targets pathological angiogenesis and lymphangiogenesis. JExp Med. 2009;206(5):1089-1102.

103. Forget MA, et al. Macrophage colony-stimulating factor augments Tie2-expressing monocyte differentiation, angiogenic function, and recruitment in a mouse model of breast cancer. PLoS One. 2014;9(6):e98623.

104. Buhtoiarov IN, et al. Anti-tumour synergy of cytotoxic chemotherapy and anti-CD40 plus CpG-ODN immunotherapy through repolarization of tumour-associated macrophages. Immunology. 2011;132(2):226-239.

105.El Kasmi KC, Stenmark KR. Contribution of metabolic reprogramming to macrophage plasticity and function. Semin Immunol. 2015;27(4):267-275.

106.Colegio OR, et al. Functional polarization of tumour-associated macrophages by tumour-derived lactic acid. Nature. 2014;513(7519):559-563.

107. Coscia M, et al. Zoledronic acid repolarizes tumourassociated macrophages and inhibits mammary carcinogenesis by targeting the mevalonate pathway. JCell Mol Med.2010;14(12):2803-2815.

108. Roelofs AJ, Thompson K, Gordon S, Rogers MJ. Molecular mechanisms of action of bisphosphonates: current status. Clin Cancer Res. 2006;12(20 pt 2):6222s-6230s.

109. Benford HL, Frith JC, Auriola S, Mönkkönen J, Rogers MJ. Farnesol and geranylgeraniol prevent activation of caspases by aminobisphosphonates: biochemical evidence for two distinct pharmacological classes of bisphosphonate drugs. $\mathrm{Mol}$ Pharmacol. 1999;56(1):131-140.

110. Kong T, Westerman KA, Faigle M, Eltzschig HK, Colgan SP. HIF-dependent induction of adenosine $\mathrm{A} 2 \mathrm{~B}$ receptor in hypoxia. FASEB J. 2006;20(13):2242-2250.
111. Eltzschig HK, Köhler D, Eckle T, Kong T, Robson SC, Colgan SP. Central role of Sp1-regulated CD39 in hypoxia/ischemia protection. Blood. 2009;113(1):224-232.

112. Synnestvedt K, et al. Ecto-5'-nucleotidase (CD73) regulation by hypoxia-inducible factor- 1 mediates permeability changes in intestinal epithelia. JClin Invest. 2002;110(7):993-1002.

113. Eltzschig HK, et al. HIF-1-dependent repression of equilibrative nucleoside transporter (ENT) in hypoxia. J Exp Med. 2005;202(11):1493-1505.

114. Morote-Garcia JC, Rosenberger P, Nivillac NM, Coe IR, Eltzschig HK. Hypoxia-inducible factordependent repression of equilibrative nucleoside transporter 2 attenuates mucosal inflammation during intestinal hypoxia. Gastroenterology. 2009;136(2):607-618.

115. Morote-Garcia JC, Rosenberger P, Kuhlicke J, Eltzschig HK. HIF-1-dependent repression of adenosine kinase attenuates hypoxia-induced vascular leak. Blood. 2008;111(12):5571-5580.

116. Bodin P, Burnstock G. Synergistic effect of acute hypoxia on flow-induced release of ATP from cultured endothelial cells. Experientia. 1995;51(3):256-259.

117. Hasko G, Pacher P. Regulation of macrophage function by adenosine. Arterioscler Thromb Vasc Biol. 2012;32(4):865-869.

118. Csóka B, et al. Adenosine promotes alternative macrophage activation via $\mathrm{A} 2 \mathrm{~A}$ and $\mathrm{A} 2 \mathrm{~B}$ receptors. FASEB J. 2012;26(1):376-386.

119. Pinhal-Enfield G, et al. An angiogenic switch in macrophages involving synergy between Tolllike receptors 2, 4, 7, and 9 and adenosine $\mathrm{A}(2 \mathrm{~A})$ receptors. Am J Pathol. 2003;163(2):711-721.

120. Dvorak HF. Tumors: wounds that do not heal. N Engl J Med.1986;315(26):1650-1659. 